\title{
$\times$ Premiers cas authentiques de farcin du bœuf en Afrique Occidentale Française
}

\author{
Note préliminaire \\ par G. MEMERY, P. MORNET et A. CAMARA
}

Nous avons reconnu dans la région de Dakar une affection qui ne semble pas avoir été signalée auparavant en A.O.F. : le farcin du beuf.

\section{I. - RAPPEL HISTORIQUE}

Le farcin du bouf, maladie infectieuse, contagieuse, inoculable, évoluant le plus souvent sous forme chronique, est dû essentiellement - à un germe de la famille des Actinomycétacées : Nocardia farcinica (1). Il se caractérise cliniquement par une inflammation suppurative des vaisseaux et des ganglions lymphatiques de la racine des membres et des membres, qui se manifeste sous la forme de cordes avec nodules, de tumeurs circonscrites et d'adénopathies. On assiste parfois à la généralisation du processus avec apparition de pseudo-tubercules sur les différents viscères et les ganglions lymphatiques.

Décrite pour la première fois en France par Sorillon en 1829, elle donne lieu au siècle dernier à de nombreuses observations et à d'importants travaux. A la même époque (1830), elle est rencontrée à la Guadeloupe où Couzin l'étudie en 1879 , en relation avec Nocard qui réussit à isoler l'agent causal. Bernard, en 1924, souligne la gravité de la maladie dans cette île.

Un bref rappel de la répartition géographique montre qu'elle est, actuellement, surtout répandue dans les pays tropicaux et subtropicaux.

En effet, en France, elle n'a plus été diagnostiquée depuis 1891 (Moussu).

(1) D'autres germes, les uns gram négatifs et acido-résistants, les autres voisins du bacille de Preisz Nocard, ont été incriminés.

Le germe Nocardia est lui-même en cours de révision avec les genres Mycobacterium et Streptomyces, une étude taxonomique étant entreprise dans les Laboratoires du Dr Waksman à l'Université d'Etat de Rutgers (U.S.A.).
Par contre, elle est signalćc en Indc ct cn Indonésie. D'autre part, la Guadeloupe en est toujours largement infectée et récemment (Gonzalo Luque, 1946), son existence a été signalée en Colombie.

En Afrique, elle sévit en Somalie, en Erythrée, au Kenya et elle est connue des éleveurs de la République du Soudan. Elle est également rencontrée à l'Ile Maurice.

En A.E.F., certains cas ont été reconnus sur des animaux abattus pour la boucherie; mais, en A.O.F., elle était, jusqu'à ces derniers temps, passée inaperçue et c'est seulement en 1957 qu'elle est décelée dans un des territoires, le Sénégal.

Nous en donnerons brièvement la relation.

\section{II. - LA MALADIE DANS LA RÉGION DE DAKAR}

Les premiers cas authentiques sont relevés sur un troupeau de bovins de la banlieue dakaroise (1). Une enquête ultérieure, effectuée dans plusieurs villages, indique 28 malades sur 576 bovins visités, appartenant à divers propriétaires.

\section{Symptômes.}

L'attention du propriétaire est attirée par un empâtement ganglionnaire qui s'accentue très lentement. Il faut noter une prédominance de l'atteinte des ganglions préscapulaires et précruraux. Un seul ou plusieurs de ces ganglions (parfois les quatre) peuvent être atteints, à des

(1) Nous remercions vivement $M$. Diallo Djibril, vétérinaire africain de la Circonscription de Dakar, qui a bien voulu nous consulter et nous donner toutes facilités de recherche. 
stades divers. Ils s'indurent, deviennent irréguliers, polynodulaires, pour atteindre la grosseur des deux poings et parfois celle de la tête d'un enfant ; ils sont alors généralement entourés de nombreux nodules de la grosseur d'une noix à celle d'un œuf. Des points fluctuants peuvent apparaitre, mais l'abcédation n'est pas de règle et est toujours tardive. Quand elle se produit, on note l'écoulement, peu abondant, d'un pus blanc, crémeux, parfois grumeleux, toujours inodore. La fistule a tendance à se refermer rapidement. La peau devient dure et adhérente à la lésion ganglionnaire sous jacente.

De ces lésions peuvent partir des cordes lymphatiques avec des nodules en chapelet qui atteignent parfois le pli du genou et descendent sur la face interne du canon. Elles sont cependant peu nombreuses et souvent courtes, même avec des lésions ganglionnaires très anciennes. Nous n'avons pas noté d'ulcérations des cordes ou des nodules qui les accompagnent.

\section{Évolution.}

La maladie est essentiellement chronique. Nous n'avons pas observé les formes aiguës ou sub-aiguès décrites à la Guadeloupe. L'évolution est très lente, un an et certainement plus. Cette durée est difficile à préciser (1). En effel, le début est très insidieux et lorsque la maladie est découverte, elle évolue depuis plusieurs semaines. L'état général des animaux atteints ne semble pas affecté, du moins au cours des premiers mois; même porteurs de lésions importantes, ils ne sont pas en plus mauvais état que les autres. Les phases ultimes, décrites par Daubney au Kenya, n' ont pas le temps de se manifester, les propriétaires se débarrassant des malades dès que les lésions sont trop apparentes.

\section{Lésions.}

\section{LÉSIONS SUPERFICIELLES.}

Au niveau des ganglions atteints, la peau est épaissie, fibreuse, lardacée, surtout lorsqu'il y. a eu abcédation et il peut se former une plaque indurée de plusieurs décimètres de diamètre. Là coque de l'abcès est toujours épaisse et fibreuse. Les abcès sont polyfocaux et contiennent un pus de consistance' variable. Il est crémeux,

(I) D'après l'enquête à laquelle nous nous sommes livrés, il semble que les premiers cas observés par nous dataient de plusieurs mois. bien lié, homogène, parfois légèrement verdâtre ou jaune, toujours inodore et n'a pas tendance à s'écouler. Mais la consistance semble dépendre du degré d'évolution de l'abcès. En effet, dans les lésions anciennes, le pus est plus liquide, moins homogène, grumeleux. Généralement, le ganglion n'est pas atteint dans sa totalité; il reste toujours une partie qui, macroscopiquement, paraît saine.

Les cordes sont constituées par les vaisseaux lymphatiques enflammés. Leur paroi est épaisse et indurée. Ils contiennent des quantités variables de pus qui s'amasse par endroit pour former les nodules en chapelet, rencontrés de loin en loin, et dont la paroi est habituellement plus mince.

\section{LESIONS PRCFONDES.}

Nous avons rencontré des adénites internes purulentes, axillaires et iliaques externes. Les ganglions peuvent atteindre le volume du poing, avec plusieurs foyers purulents de même nature que précédemment.

Ils sont reliés aux ganglions externes par des trajets purulents qui suivent le chemin des lymphatiques profonds.

$\mathrm{Au}$ point de vue viscéral, nous avons noté à deux reprises de petites lésions pulmonaires mais les ganglions bronchiques et médiastinaux étaient normaux.

Nous n'avons pas constaté à Dakar les importantes lésions viscérales signalées par Bernard à la Guadeloupe, et par divers auteurs en Inde.

\section{III. - LA MALADIE DANS LA RÉGION DE THIES ET LA PETITE COTE (1)}

A l'occasion d'une visite au Centre de l'Elevage de Thiès (2) en novembre 1957, l'un d'entre nous note qu'un bœuf producteur de sérum antipestique $\left(n^{\circ} 1\right)$, isolé du reste du troupeau le 17 octobre, présente un engorgement du ganglion lymphatique préscapulaire droit avec une amorce de corde lymphatique.

Mais le signe le plus frappant est une lymphangite tronculaire du membre postérieur droit.

(1) On appelle, au Sénégal, "Petite Côte ", la région littorale qui s'étend au sud de Dakar, vers M'Bour, Joal.

(2) Ville située à $70 \mathrm{~km}$ au nord-est de Dakar. 
Le rapprochement avec les observations de Dakar est immédiat et la suspicion de farcin s'impose. Mais avant de faire la description de ce cas très intéressant, nous relaterons ce qu'une première enquête nous a appris sur cette affection dont l'existence semble confirmée, depuis plusicurs années, dans les régions de Thiès, M'Bour, Joal. peutique mise en œuvre n'empêche pas les lésions de progresser et une lymphangite tronculaire massive envahit le membre intéressé.

Une dermite compliquée de plaie infectée s installe au niveau du métatarse. Et la suppuration gagne, en position déclive, la couronne des deux onglons. L'amaigrissement s'installe,

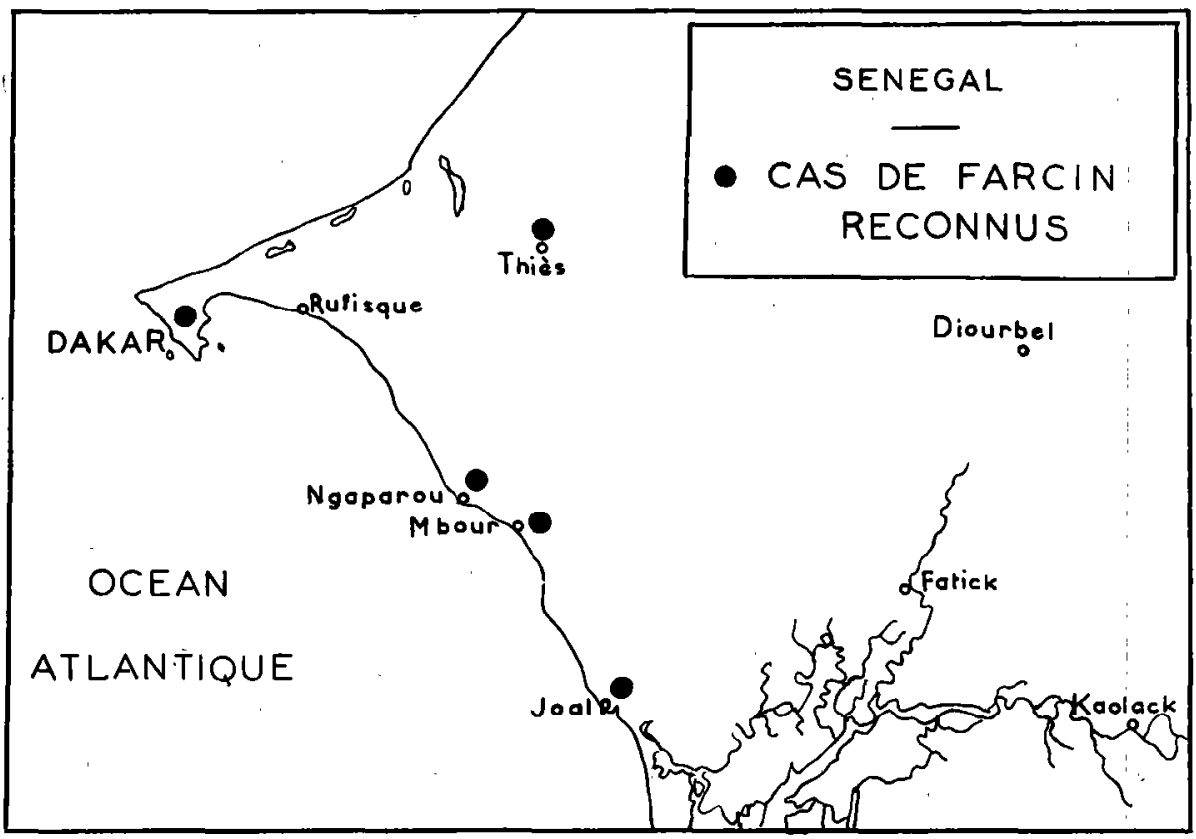

En 1956, le rapport d'un agent du Service de l'Elevage fait état d'une affection, dénommée "Boudel " (1) par les éleveurs. Elle aurait été assimilée par lui, non sans quelque raison apparente, à la lymphangite épizootique du cheval. Les traitements habituels à cette demière maladie auraient été essayés : biodure dc mercure et lugol. Sans résultat.

Des cas de "Boudel " seraient également signalés de façon sporadique dans les régions de M'Bour, N'Gaparou, Joal.

Mais revenons au foyer de farcin constitué par le troupeau de bovins producteurs de sérum antipestique du Centre de Thiès.

Le 10 septembre 1957, un bœuf $\mathrm{n}^{\mathrm{o}} 11$ est isolé parce qu'il présente une corde lymphatique avec " boutons " non ouverts le long de la saphène externe du membre postérieur droit. La théra-

(1) Le fait que le farcin soit désigné par un nom vernaculaire indique bien l'ancienneté de l'affection. des adénopathies préscapulaires apparaissent, importantes, et l'animal meurt dans le marasme le 25 septembre 1957.

Le bœuf $n^{0} 1$ (cité plus haut) présente les mêmes lésions que le $n^{0} 11$ et l'évolution se poursuit de façon superposable.

Cet animal est transporté au Laboratoire Central de l'Elevage à Dakar, le 23 décembre, pour un cxamen plus approfondi. L'amaigrissement s'intensifie et, le 7 janvier 1958, il est sacrifie in extremis.

L'autopsie révèle des lésions très étendues et très instructives. En voici la relation :

État général : très mauvais : hydrocachexie marquée. Hypertrophie nette du ganglion poplité droit, des ganglions préscapulaires droit et gauche avec nodules périphériques.

\section{Lésions.}

MEMBRE POSTÉRIEUR DROIT : le membre " éléphantiasique" montre une peau épaissie, 
fibro-lardacée, adhérente au tissu sous-jacent. Tous les lymphatiques sont hypertrophiés, remplis d'un caseum jaunâtre avec concrétions calcaires. Les traînées lymphatiques longitudinales, semées de caseum, donnent un aspect très particulier au membre dépouillé.

Grandes Cavités : légère pleurésie séreuse du côté droit.

APPAREIL RESPIRATOIRE : collapsus pulmonaire à droite; broncho-pneumonie nodulaire caséeuse ou caséo-calcaire, disséminée dans tout le parenchyme. La grosseur des nodules varie de la tête d'une épingle à celle d'un ouf de pigeon:

APPAREIL DIGESTIF : légère entérite ; nodules parasitaires casćeux sous-muqueux de l'intestin grêle (œsophagostomose); présence de nodules caséeux à Nocardia de la taille d'un grain de mil, disséminés dans le foie.

ApPareil Circulatoire : phlébite de la jugulaire gauche (accidentelle : consécutive à une injection médicamenteuse irritante). Thrombose récente, mais en voie d'organisation, de la veine fémorale droite. Phlébite et périphlébite caséeuse au voisinage du ganglion poplité.

APPAREIL LYMPHOPOÏÉTIQUE : nodules caséeux ou caséo-calcaires du volume d'une tête d'épingle dans le parenchyme splénique. Présence de nodules caséeux ou caséo-calcaires dans les ganglions suivants :

- poplités : le ganglion gauche est peu lésé rrais le droit, en relation avec le membre "éléphantiasique ", est très hypertrophié (de la grosseur d'une pomme), entièrement envahi par un caséum qui en a détruit la substance, et a débordé sur les parties musculaires périphériques, créant une myosite nodulaire caséocalcaire ;

- inguinal droit, iliaque droit, pancréatique, trachéobronchiques et médiastinaux, rétro-pharyngien droit, parotidien gauche.

\section{IV. - L'AGENT CAUSAL}

Siège du microorganisme dans les lésions.

L'agent peut aisément passer inaperçu; il est en effet pratiquement absent dans le pus de lésions anciennes (foyer de ramollissement par exemple). Il est abondant à la périphérie des lésions ou au centre des microabcès (lésions débutantes).
La meilleure méthode de coloration pour le mettre en évidence sur un frottis de matériel pathologique est la méthode de Ziehl à condition de ne pas trop décolorer à l'alcool. Le germe se manifeste sous l'aspect de petites colonies mycéliennes ramifiées en étoile ou en "araignée ", rouges sur fond bleu.

\section{Isolement.}

Nous avons toujours isolé facilement le germe en nous adressant toutefois à des lésions peu anciennes. Nous avons employé le milieu de Lôwenstein sur lequel le développement est le plus rapide et le plus abondant, cependant que les souillures sont généralement inhibées. En 4 à 5 jours, de petites colonies pigmentées en jaune beurre apparaissent. Elles se développent le plus souvent sous forme $\mathrm{R}$, chagrinées; à surface irrégulière et crevassée. La pigmentation s'accentue, devient ocre jaune et diffuse dans le milieu qui perd sa couleur initiale et devient également ocre jaune. Parfois, les colonies sont moins sèches, légèrement brillantes et lisses, avec une partie centrale surélevée, entourée d'un bourrelet marginal très net.

L'isolement peut aussi être fait sur milieu ordinaire, mais la culture est très longue à apparaître, quinze jours, parfois trois semaines, et risque d'être envahie par les cultures de germes ou de champignons secondaires qui souillent souvent les prélèvements.

\section{Cultures du microorganisme dans les milieux usuels.}

En gélose nutritive ordinaire; il pousse très lentement en donnant, en 8 à 10 jours, de petites colonies atteignant $2 \mathrm{~mm}$ de diamètre, chagrinées, irrégulières, mais toujours minces, souvent pigmentées en ocre rouge.

Sur bouillon ordinaire, on observe un voile gras et épais ne couvrant pas toute la surface du liquide. Il tombe régulièrement au fond $\mathrm{du}$ tube et donne un dépôt. Le milieu reste clair.

Sur pomme de terre, la culture est un peu plus rapide, les colonies sont confluentes, irrégulières, et très pigmentées. Le pigment diffuse dans la pomme de terre au bout d'un ou deux mois de culture.

Sur carotte, le germe pousse aussi assez abondamment mais est toujours moins pigmenté que sur pomme de terre. 
Aspect du microorganisme sur les cultures.

Il est morphologiquement différent de celui noté dans les prélèvements pathologiques. Il se présente sous forme de filaments enchevêtrés. Certaines souches donnent des bacilles isolés ou un strepto-bacille avec des amas d'éléments cocciformes de grosseur très variable.

\section{Affinités tinctoriales.}

Il se colore très bien au bleu de méthylène à 1 p. 100 . Il présente alors un grand nombre de granulations métachromatiques, qui sont aussi présentes dans les bacilles isolés ; certaines sont même à l'état libre, et constituent les éléments cocciformes signalés.

Le germe prend le gram si la décoloration à l'alcool est modérée. Il est uniformément coloré.

Il est acido-alcoolo-résistant; il se laisse cependant décolorer par un excès d'alcool. Et au Ziehl, une partie des germes sont souvent colorés en bleu.

\section{V. - DISCUSSION}

Le farcin du bœuf, tel que nous le décrivons, ne constitue pas une entité différente de celle connue en d'autres régions.

Mais il est intéressant d'approfondir divers problèmes s'y rapportant car cette affection est économiquement grave, d'une chronicité décevante et d'une guérison problématique.

Et si, du vivant de l'animal, le diagnostic en est relativement facile (lésions externes) pour un clinicien averti, il n'en est pas de même sur l'animal abattu, lors de lésions viscérales. C'est ainsi que dans le cas du høuf $\mathrm{n}^{0} 1$ (Thiès), il était impossible de différencier les lésions de celles de la tuberculose. Seuls l'examen microscopique et la culture du germe purent lever les doutes.

Et cette confusion plausible pose pour l'inspection des viandes un problème très sérieux, les mesures sanitaires étant, en ce qui concerne l'infection à Bacille de Koch, très différentes de celles prévues pour le farcin.

Dans l'observation que nous relatons, peutêtre un spécialiste aurait-il pu s'étonner de la discrétion des lésions de la plèvre dans une infection tuberculeuse, eu égard à l'importance des lésions bronchopulmonaires.

Quoi qu'il en soit, actuellement, une enquête s impose pour déterminer l’importance de la maladie farcineuse, son extension, sa répartition et rechercher la part respective qui revient, en certaines régions, à la tuberculose et au farcin.

\section{VI. - CONCLUSION}

Nous décrivons les premières observations authentiques dc farcin du bøuf relevćes cn Afrique Occidentale Française (territoire du Sénégal). L'existence de cette maladie dans la région de la petite côte du Sénégal semble déjà ancienne.

Est-ellc passéc inaperçue ou l'a-t-on confondue avec une autre maladie? Nous ne pouvons donner de réponse à cette question.

Nous soulignons la difficulté, dans certains cas, d'assurer le diagnostic différentiel du farcin et de la tuberculose sans le secours du laboratoire.

La symptomatologie, l'évolution de la maladie, l'étude des lésions et du germe, Nocardia farcinica, sont exposées sommairement.

Une étude plus approfondie portant sur l'extension de la maladie, le pouvoir pathogène du germe sur animaux d'expérience, l'histopathologie et le résultat des divers traitements entrepris sera publiée ultérieurement.

(Laboratoire Central de l'Elevage " Georges CURRASSON" à Dakar. Directeur : P. Mornet.) 